\title{
Determinación de la habilidad cognitiva en la resolución de problemas matemáticos de los estudiantes de Ciencias Empresariales de la Universidad Francisco de Paula Santander
}

Juan Simón Jaimes Boada ${ }^{1}$

\section{Resumen}

La Constitución Política de 1991 en Colombia generó la revolución educativa, encontrando políticas cada vez más adecuadas y eficaces que se ajusten a la realidad institucional del país, y que promuevan el mejoramiento del sistema hacia la calidad, la eficiencia, la equidad y su pertinencia. Teniendo el Estado mecanismos para evaluar, como es, a nivel superior los exámenes de calidad de la educación superior (ECAES). Por lo tanto, es responsabilidad de las instituciones educativas, a partir de los resultados obtenidos, implementar planes de mejoramiento, para afianzar las fortalezas y disminuir las debilidades. Lo anterior concreta la presente investigación de campo, de carácter descriptivo, en determinar la habilidad cognitiva en la resolución de problemas matemáticos de los estudiantes de ciencias empresariales de la Universidad Francisco de Paula Santander. Para dar cumplimiento a lo propuesto, la investigación se apoya en tres teorías: La teoría triárquica de la inteligencia humana de Sternberg, la teoría de los campos conceptuales de Vergnaud y la teoría de las fases de resolución de problemas de Polya. Permitiendo desarrollar cuatro pruebas: C1, conocimientos en la compresión lectora, C2, conocimientos en la selección del plan, C3, conocimientos en la selección de estrategias y C4, conocimientos en la ejecución del plan de trabajo; aplicados en forma consecutiva a una muestra de 75 estudiantes de Administración y Contaduría de tercer y cuarto semestre. Se realizó análisis de confiabilidad y estudio psicométrico a cada una de ellas y en forma global de aciertos y continuidad de las fases del proceso de resolución mencionadas anteriormente; así, como las opiniones de algunos alumnos participantes en la prueba. Con el anterior estudio, se demuestra que los estudiantes no llevaron a cabo todo el proceso de las cuatro fases en las diferentes situaciones planteadas; en la fase tres, donde más avanzaron fue el criterio diez, con el 12 por ciento y en la fase 2 el 21 por ciento. Además, presentan debilidad en la comprensión lectora y en análisis de problemas que requieren una fortaleza conceptual. Por tal motivo se presenta una propuesta metodológica conducente a mejorar las debilidades encontradas y además permita realizar futuras investigaciones en este campo.

Palabras claves: Calidad, fortalezas, debilidades, habilidad, conocimiento, aprendizaje.

${ }^{1}$ Universidad Francisco de Paula Santander, Adscrito al departamento de Matemáticas y Estadística. Correo: juansi_57@hotmail.com 


\section{Abstract}

The Constitution of 1991 in Colombia led the revolution in education, increasing political finding appropriate and effective to meet the country's institutional reality, and promote improvement of the system to quality, efficiency, equity and relevance. Taking the State mechanisms to assess, as it is, a higher level of quality reviews of higher education (ECAES). Therefore, it is the responsibility of educational institutions, from the results, implement improvement plans to enhance the strengths and minimize weaknesses. This particular field of this research, descriptive, to determine cognitive ability in solving mathematical problems of business students at the University Francisco de Paula Santander. To comply with the proposed, the research is based on three theories: The theory of human intelligence triarchic Sternberg's theory Vergnaud's conceptual fields and the theory of the stages of Polya's problem-solving. Possible to develop four tests: C1, knowledge in reading comprehension, $\mathrm{C} 2$, expertise in selecting the plan, $\mathrm{C} 3$, knowledge in the selection of strategies and $\mathrm{C} 4$, knowledge in implementing the work plan; applied consecutively to a sample of 75 students of Administration and Accounts of the third and fourth semester. The analysis of the reliability and psychometric study each of them and holistic approach to success and continuity of the phases of the aforementioned resolution, as well as the views of some students participating in the test. The previous study shows that students did not perform the whole process of the four phases in the different situations arising, in phase three, where more advanced approach was ten, with 12 percent and in phase 221 percent. Furthermore, they have weaknesses in reading comprehension and analysis of problems requiring conceptual strength. For this reason, proposes a method conducive to improving the weaknesses found and also allow future research in this field.

Keywords: Quality, strengths, weaknesses, skill, knowledge, learning.

\section{Introducción}

E sta investigación de campo de carácter descriptivo pretende determinar la habilidad cognitiva en la resolución de problemas matemáticos de los estudiantes de ciencias empresariales de la Universidad Francisco de Paula Santander, con el propósito de formular estrategias que permitan mejorar el aprendizaje de la matemáticas, adquiriendo una mejor conceptualización para enfrentar los Exámenes de calidad de la educación superior (ECAES ) y por ende el desempeño eficaz en sus futuras profesiones.

El objetivo general se ha dirigido a determinar las habilidades cognitivas utilizadas por los estudiantes de ciencias empresariales de la Universidad Francisco de Paula Santander en la resolución de problemas matemáticos. Para ello se han elaborado cuatro pruebas $(\mathrm{C} 1$ : componentes cognitivos en la compresión lectora, C2: Componentes cognitivos en la selección del plan de trabajo, C3: Componentes cognitivos en la organización de estrategias y C4: Componentes cognitivos en la ejecución del plan de trabajo) basadas en las teorías de la inteligencia triarquica de Sternberg (1985 a), de los campos conceptuales de Vergnaud (1982) y la de las fases en la resolución de problemas de Polya (1957) y siguiendo la investigación realizada por Toboso (2004) en este campo; con la finalidad de valorar y describir la habilidad adquirida en el aprendizaje de la matemática, para llevar a cabo las cuatro etapas del proceso de resolución de problemas: Conocimiento lingüístico semántico, necesario para comprender el problema, conocimiento esquemático, que fundamenta el recono- 
cimiento de su naturaleza y la elección del plan de resolución, conocimiento estratégico, como organizador de los pasos a seguir y conocimiento algorítmico que ejecuta las operaciones finales para obtener la solución.

Además, se presenta una propuesta metodológica conducente a mejorar las debilidades encontradas en la investigación buscando un aprendizaje significativo y por otra parte conlleve a futuras investigaciones.

Desde esta perspectiva se ha planificado y desarrollado esta investigación en cinco capítulos.

En el primer capítulo se exponen las razones básicas que de conformidad con la constitución política Colombiana de 1991 generó la revolución educativa centrada en la calidad, la eficiencia, la equidad y la pertinencia. El estado realiza seguimiento, con los exámenes de calidad de educación superior (ECAES) y es responsabilidad de las instituciones educativas a partir de estos resultados elaborar planes de mejoramiento. Lo anterior concreta este estudio en la determinación de la habilidad cognitiva en la resolución de problemas matemáticos de los estudiantes de ciencias empresariales de la Universidad Francisco de Paula Santander.

En el segundo capítulo se sintetizan los sistemas de evaluación de aprendizaje tanto internacional como nacional, haciendo énfasis en los resultados de los ECAES aplicados consecutivamente desde el año 2004, donde los resultados han desmejorado año tras año. Además, de una síntesis de la investigación nacional de Hernández, R. V. ; González, R. M. ; Mendoza, S. M. ; Isidro, H .y Peñaranda N. B. (2006). E internacionales de Valle Espinosa M. C. Juárez Ramón M. y .Guzmán Ovando M. E. (2007) Y Toboso, J. (2004).

También, se exponen los fundamentos teóricos de la teoría triarquica de la inteligencia humana de Sternberg (1985 a, 1985b, 1986, 1965 a, 1986 y 1987), Prieto y Sternberg (1990), Serrano (1994), Sternberg y Rifkin (1979), fundamentada en el acto inteligente y la resolución de problemas desde la triple concepción jerárquica en la que intervienen los componentes cognitivos, la experiencia y el contexto de la persona.
A continuación se presenta la teoría de los campos conceptuales de Vergnaud (1982, 1983 a, 1983 b, 1988, 1990, 1993, 1994, 1996 a, 1996 b, 1997, 1998), Moreira (2002) y Lester (1983), fundamentada en que el conocimiento esta organizado por campos conceptuales y su aprendizaje requiere de un largo periodo de tiempo que depende de la experiencia y la instrucción del individuo.

Finalmente, la teoría de las fases de resolución de problemas realizada por Polya (1957), influenciado por las ideas del modelo Gestaltista y basándose en sus observaciones directas como profesor de matemáticas, considera que son necesarias las siguientes fases: Comprensión del problema, elaboración del plan, puesta en marcha del plan y la visión retrospectiva o reflexión.

En el tercer capítulo se fundamenta la modalidad de investigación de campo y de carácter descriptivo de esta investigación, según el Manual de la UPEL, Ary (1989) y Tamayo y Tamayo (2001). Luego se explica la estructura del diseño de las cuatro pruebas para ser aplicadas a una muestra de 75 estudiantes de una población de 330 (30 estudiantes de Administración de Empresas y 45 de Contaduría Pública) y las técnicas de procesamiento y análisis de información.

El cuarto capítulo se presenta los análisis e interpretación de los resultados como son la confiabilidad y el estudio spicométrico de las cuatro pruebas que permiten cumplir con los objetivos de la investigación. El capítulo $\mathrm{V}$ recoge las conclusiones, recomendaciones y la propuesta metodológica y finalmente la referencia bibliográfica y los anexos elaborados para realizar esta investigación.

\section{Análisis e Interpretación de Resultados}

Se analiza la confiabilidad y los aspectos básicos de las características spicométricas para cada uno de las cuatro pruebas desarrolladas en la investigación (C1, C2, C3, y C4). Para la confiabilidad se aplica el método de las mitades partidas o splitthalves.

En el análisis psicométrico se presentan en primer lugar las medias y desviaciones típicas de cada uno 
de los ítem: Estos datos ponen de relieve el grado de dificultad que han encontrado los alumnos en los problemas planteados (Toboso, pp 195). Facilitando la toma de decisiones en la reorientación del proceso enseñanza aprendizaje de las matemáticas en Ciencias Empresariales de La Universidad Francisco de Paula Santander.

Después se presenta los resultaos obtenidos en cada uno de los distractores, los porcentajes de error y acierto en cada ítem de cada una de las cuatro pruebas, por carrera y en total. Y, al final los resultados respecto a la continuidad de las etapas del proceso en la resolución de problemas matemáticos de cada uno de los participantes, lo cual nos facilitará la toma de decisiones que lleven a intensificar y reforzar los contenidos para un mejor aprendizaje significativo de las matemáticas.

A continuación de los análisis anteriores se registran las opiniones de algunos estudiantes participantes en la prueba, a quienes se les interrogó sobre la dificultad, la importancia y la pertinencia de la prueba con respecto a los contenidos vistos en las asignaturas de matemáticas I y matemáticas II de Administración de Empresas y Contaduría Pública.

A continuación se presenta algunas tablas y gráficos tenidos en cuenta.

Tabla 1. Estadísticos descriptivos de la prueba C1

\begin{tabular}{|c|c|c|c|c|c|}
\hline Ítem & Media & $\begin{array}{c}\text { Des. } \\
\text { Tip. }\end{array}$ & Ítem & Media & $\begin{array}{c}\text { Des. } \\
\text { Tip. }\end{array}$ \\
\hline C1.1 &, 31 &, 464 & $\mathrm{C} 1.6$ &, 57 &, 498 \\
\hline $\mathrm{C} 1.2$ &, 24 &, 430 & $\mathrm{C} 1.7$ &, 09 &, 293 \\
\hline $\mathrm{C} 1.3$ &, 36 &, 483 & $\mathrm{C} 1.8$ &, 13 &, 342 \\
\hline $\mathrm{C} 1.4$ &, 32 &, 470 & $\mathrm{C} 1.9$ &, 20 &, 403 \\
\hline $\mathrm{C} 1.5$ &, 61 &, 490 & $\mathrm{C} 1.10$ &, 36 &, 483 \\
\hline
\end{tabular}

Tabla 2. Distractores Ítem 1.1

\begin{tabular}{|c|c|c|c|c|c|c|}
\hline \multirow{2}{*}{ ITEM 1.1 } & \multicolumn{2}{|c|}{ Frecuencia } & \multicolumn{2}{c|}{ Porcentaje } & \multicolumn{2}{c|}{ TOTAL } \\
\cline { 2 - 7 } & $\mathrm{A}$ & $\mathrm{C}$ & $\mathrm{A} \%$ & $\mathrm{C} \%$ & & $\%$ \\
\hline NO CONTESTA & & & & & & \\
\hline a) Error & 5 & 1 & 16.7 & 2.2 & 6 & 8 \\
\hline b) Error & 14 & 27 & 46.7 & 60 & 41 & 54.7 \\
\hline c) Error & 1 & 4 & 3.3 & 8.9 & 5 & 6.7 \\
\hline d) Acierto & 10 & 13 & 33.3 & 28.9 & 23 & 30.6 \\
\hline Total & 30 & 45 & 100 & 100 & 75 & 100 \\
\hline
\end{tabular}

resultados item 1.1

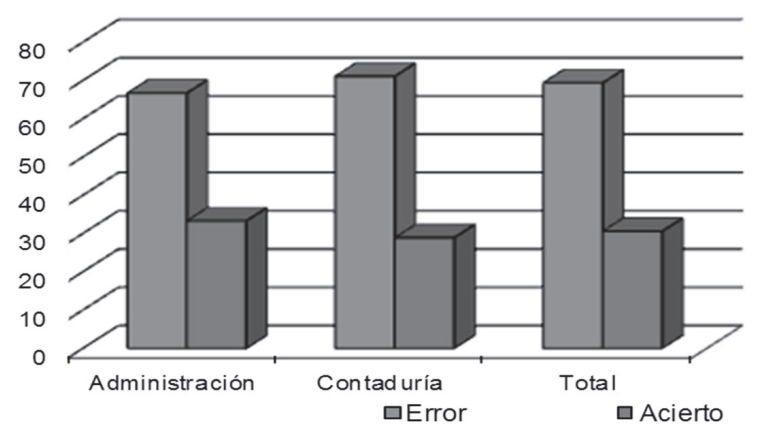

Figura 1. Resultados de la prueba C1. Ítem 1.1.

Tabla 3. Sumatorias de los aciertos obtenidos en cada uno de los ítem de las cuatro pruebas

\begin{tabular}{|c|c|c|c|c|c|c|c|c|c|}
\hline \multirow{2}{*}{ Ítem } & \multirow{2}{*}{$\begin{array}{c}\text { Criterio de } \\
\text { evaluación }\end{array}$} & \multicolumn{9}{|c|}{ Pruebas } \\
\cline { 3 - 11 } & & \multicolumn{2}{|c|}{ C1 } & \multicolumn{2}{c|}{ C2 } & \multicolumn{2}{c|}{ C3 } & \multicolumn{2}{c|}{ C4 } \\
\cline { 3 - 11 } & & $\mathrm{A}$ & $\mathrm{C}$ & $\mathrm{A}$ & $\mathrm{C}$ & $\mathrm{A}$ & $\mathrm{C}$ & $\mathrm{A}$ & $\mathrm{C}$ \\
\hline 1 & 1 & 10 & 13 & 21 & 38 & 3 & 3 & 18 & 40 \\
\hline 2 & 2 & 9 & 9 & 11 & 19 & 13 & 21 & 1 & 27 \\
\hline 3 & 3 & 10 & 17 & 6 & 5 & 7 & 14 & 6 & 6 \\
\hline 4 & 4 & 9 & 15 & 24 & 26 & 4 & 6 & 3 & 3 \\
\hline 5 & 5 & 18 & 28 & 13 & 31 & 10 & 18 & 0 & 6 \\
\hline 6 & 6 & 15 & 28 & 9 & 15 & 4 & 5 & 3 & 11 \\
\hline 7 & 7 & 4 & 3 & 14 & 17 & 6 & 24 & 0 & 1 \\
\hline 8 & 8 & 6 & 4 & 4 & 4 & 12 & 18 & 0 & 0 \\
\hline 9 & 9 & 5 & 10 & 13 & 8 & 15 & 27 & 0 & 0 \\
\hline 10 & 10 & 9 & 18 & 15 & 27 & 10 & 19 & 0 & 0 \\
\hline
\end{tabular}

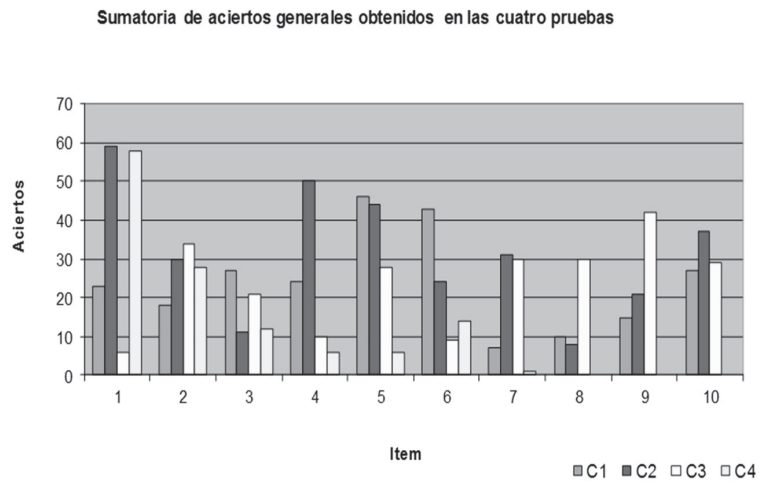

Figura 2. Sumatoria de los aciertos obtenidos en las cuatro pruebas

Tabla 4. Continuidad de las cuatro etapas del proceso de solución de problemas

\begin{tabular}{|c|c|c|c|c|c|c|c|c|c|c|c|c|}
\hline \multirow{2}{*}{$\begin{array}{c}\text { Criterios de } \\
\text { evaluación }\end{array}$} & \multicolumn{2}{|c|}{$\begin{array}{c}\text { Etapas } \\
\text { 1 a 2 }\end{array}$} & \multicolumn{2}{|c|}{$\begin{array}{c}\text { Etapas } \\
\text { 1 a 3 }\end{array}$} & \multicolumn{2}{c|}{$\begin{array}{c}\text { Etapas } \\
1 \text { a 4 }\end{array}$} & \multicolumn{2}{c|}{$\begin{array}{c}\text { Etapas } \\
\text { 2 a 3 }\end{array}$} & \multicolumn{2}{c|}{$\begin{array}{c}\text { Etapas } \\
\text { 2 a 4 }\end{array}$} & \multicolumn{2}{|c|}{$\begin{array}{c}\text { Etapas } \\
\text { 3 a 4 }\end{array}$} \\
\cline { 2 - 15 } & $\mathrm{A}$ & $\mathrm{C}$ & $\mathrm{A}$ & $\mathrm{C}$ & $\mathrm{A}$ & $\mathrm{C}$ & $\mathrm{A}$ & $\mathrm{C}$ & $\mathrm{A}$ & $\mathrm{C}$ & $\mathrm{A}$ & $\mathrm{C}$ \\
\hline 1 & 5 & 11 & 1 & 0 & 0 & 0 & 1 & 3 & 0 & 3 & 1 & 3 \\
\hline 2 & 3 & 4 & 2 & 1 & 0 & 0 & 7 & 9 & 1 & 6 & 1 & 13 \\
\hline 3 & 3 & 1 & 1 & 0 & 0 & 0 & 2 & 1 & 0 & 0 & 1 & 4 \\
\hline 4 & 8 & 9 & 1 & 1 & 0 & 0 & 3 & 2 & 1 & 0 & 1 & 1 \\
\hline 5 & 10 & 20 & 2 & 4 & 0 & 0 & 4 & 12 & 0 & 0 & 0 & 0 \\
\hline 6 & 5 & 10 & 1 & 0 & 0 & 0 & 1 & 1 & 0 & 0 & 0 & 1 \\
\hline 7 & 1 & 2 & 0 & 1 & 0 & 0 & 3 & 10 & 0 & 0 & 0 & 1 \\
\hline 8 & 1 & 1 & 0 & 0 & 0 & 0 & 2 & 2 & 0 & 0 & 0 & 0 \\
\hline 9 & 0 & 3 & 0 & 2 & 0 & 0 & 5 & 5 & 0 & 0 & 0 & 0 \\
\hline 10 & 5 & 11 & 3 & 6 & 0 & 0 & 8 & 13 & 0 & 0 & 0 & 0 \\
\hline
\end{tabular}


Continuidad de las etapas del proceso de solución de problemas

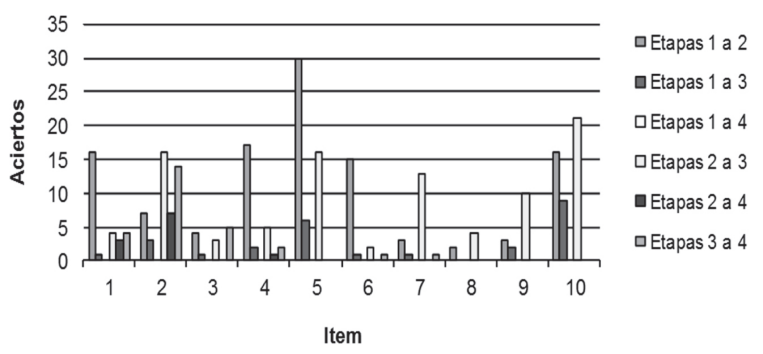

Figura 3. Continuidad de las etapas del proceso de solución de problemas.

\section{Conclusiones y Recomendaciones}

De acuerdo al análisis realizado y con base en los objetivos propuestos, se llegó a las siguientes conclusiones y recomendaciones:

El primer objetivo específico proponía valorar las habilidades cognitivas de los estudiantes de ciencias empresariales de la Universidad Francisco de Paula Santander en la resolución de problemas matemáticos, según las teorías propuestas por Sternberg, Vergnaud, Polya y otros, donde se pudo discernir que los estudiantes no llevaron a cabo todo el proceso en la solución de los diferentes problemas planteados en la prueba, es decir cumplir con el desarrollo de las cuatro etapas secuénciales planteadas por los investigadores antes mencionados.

Tomando como referencia el ítem o criterio de evaluación donde más avanzaron los estudiantes de la etapa uno a la tres, se puede observar que el 12 por ciento desarrollaron hasta la etapa tres del ítem 10 correspondientes al criterio de optimización de funciones. Y, observando el mismo ítem 10 se puede concluir que el 21 por ciento había llegado hasta la etapa dos. De la misma forma se pueden analizar cada uno de los otros ítems o criterios de evaluación que conformaban la prueba.

Además, se puede concluir también, que aunque teniendo muy débil las etapas anteriores a la ejecución del plan, algunos estudiantes pudieron resolver los problemas como es el caso de los ítem 1 y 2 con 58 y 28 estudiantes respectivamente, correspondientes a los criterios de interés simple, despeje de la incógnita de la formula planteada y progresiones aritméticas, debido a que la exigencia conceptual no era tal alta, sino que se centraba en un desarrollo mecánico de una fórmula..

Con lo anterior, podemos concluir que los alumnos muestran debilidades en la resolución de problemas matemáticos. Según opinión de los estudiantes lo anterior puede deberse a que no están adquiriendo un conocimiento significativo sino que están estudiando sólo por el momento con el fin de pasar la materia es decir adquiriendo un conocimiento memorístico para enfrentar la evaluación momentánea y dan por terminado el proceso de aprendizaje.

Por lo tanto es recomendable que la Universidad a través del departamento de matemáticas y Estadística genere estrategias para que los profesores encargados de las asignaturas de matemáticas en los programas de administración y contaduría tengan reuniones más frecuentes y entre ellos propongan metodologías y prácticas pedagógicas comunes y concertadas, con seguimiento en desarrollo de contenidos, tendientes a conseguir aprendizajes más significativos en el estudiante..

El segundo objetivo específico planteaba describir las habilidades cognitivas que presentan los estudiantes de ciencias empresariales de la Universidad Francisco de Paula Santander, teniendo en cuenta que en el proceso del aprendizaje de las matemáticas la resolución de problemas es el fin optimo al que debe llegar un estudiante para que su desempeño profesional sea de calidad.

Se puede discernir, a partir de los resultados obtenidos, que existen debilidades en la forma como se están apropiando del conocimiento matemático, en primer lugar por la baja comprensión de lectura que presenta los estudiantes. En la mayoría de los problemas planteados la comprensión de lectura estuvo por debajo de los resultados de las otras tres etapas (selección del plan de trabajo, organización de estrategias y ejecución del plan de trabajo), la mejora puede deberse en que los estudiantes muestran habilidad en el manejo de formulas (proceso mecánico) es decir, a partir de una formula identifican la variable y la despejan correctamente; pero, en problemas que deben reflexionar y en donde a partir de un desarrollo cognitivo presentar un plan de solución, la habilidad 
cognitiva es débil y no pueden enfrentar con eficiencia la resolución de los problemas, como son los casos de los ítem 7, 8, 9 y 10 correspondientes a los criterios de evaluación de oferta y demanda, curvas de nivel, análisis marginal y optimización de funciones respectivamente.

Las debilidades encontradas en forma general, se reflejan en cada una de las carreras de Administración y Contaduría similarmente, por cuanto los resultados son del mismo orden como se observa en las gráficas de análisis de aciertos, donde además, se encontró que el porcentaje de aciertos estuvo por debajo del porcentaje de error, en la mayoría de los ítem de las pruebas, con diferencias considerables.

Según Vergnaud (1996 a, p 118) el desarrollo cognitivo se obtiene con la conceptualización, piedra angular de la cognición (1998, p 173). Luego se debe prestar toda la atención a los aspectos conceptuales de los esquemas y análisis conceptual de las situaciones. En este sentido, es recomendable presentar a los alumnos en cada uno de los campos conceptuales tratados, situaciones o problemas que les permitan afianzar la conceptualización, a través de los esquemas y los invariantes (teoremas y conceptos) y logren alcanzar el desarrollo cognitivo significativo y no memorístico que les permita enfrentarse con eficacia en el desempeño de sus perfiles profesionales.

La confiabilidad con valores de 67.02, 67.5, 60.6 y 60.4 en cada una de las pruebas, es satisfactoria para esta investigación y de los análisis de distractores se encuentra que estos cumplen con su objetivo en todos los ítems. Se recomienda para una próxima aplicación, mejorar aquellos que están por el orden del tres por ciento.

En vista de que los resultados muestran que el aprendizaje adquirido por los estudiantes de Administración y Contaduría en los contenidos de matemáticas para el desempeño optimo de sus profesiones es bastante débil, se presenta una propuesta con base en una guía de trabajo independiente para el estudiante con las siguientes pautas: Contenidos a desarrollar, introducción, competencias, actividades de aprendizaje, evaluación y bibliografía, con el objetivo de mejorar estas debilidades observadas y que sea punto de partida para debates, diálogos, concertaciones y otras.
Como la mayor debilidad es la comprensión de lectura la guía en las actividades de aprendizaje sólo cita el campo conceptual y el estudiante o los estudiantes (puede desarrollarse en grupo) deberán buscar el problema donde aplique la conceptualización, realizando la resolución del problema con las etapas tratadas en esta investigación y recomendada por los expertos. De esta forma se refuerza la comprensión de lectura matemática y por ende las etapas de resolución de problemas.

\section{Referencias Bibliográficas}

Ary, D. (1989). Introducción a la investigación pedagógica. México: McGraw-Hill.

Exámenes de Calidad de la Educación Superiores Administración, Marco de fundamentación conceptual, ECAES (2004), Unión temporal PRO. CAD, Bogotá D. C. [Documento en línea]. Disponible: http://www.mineducación.gov.co o www. Icfes.gov.co [Consulta: 2009, Marzo 21].

Lester, F. K. (1983). Trends and issues in mathematical problem solving research. En Lesh, R. y Landau, M. (Eds.), Acquisition of mathematical concepts and processes. New York: Academic Press.

Moreira, M. (2002) La teoría de los campos conceptuales de Vergnaud, la enseñanza de las ciencias y la investigación en el área, Instituto de Física, UFRGS, Caixa Postal 15051, Porto Alegre.

Polya, G. (1957). How to solve it. Garden City, New York: Doubleday Anchor. En castellano, Cómo plantear y resolver problemas. México: Trillas, 1987.

Serrano, FJ. (1994). Evaluación de la interacción de los estilos de enseñanza y aprendizaje en los contextos escolares. Murcia: Tesis doctoral

Sternberg, R.J. y Rifkin, B.(1979). The development of analogical reasoning processes. Jounal of Experimental Child Psychology, 27, 195-232.

Sternberg, R.J. (1985b). Human intelligence: the model is the message. Science, 230, 1111-1118.

Sternberg, R. J. (1986). Intelligence applied. Un- 
derstanding and incresing your intellectual skill. San diego, C. A.: Harcourt Brace Jovanovich.

Sternberg, R. J. (1987). Inteligencia Humana, Vol. I y II. Barcelona: Paidos Ibérica.

Tamayo y Tamayo, M. (2001) El proceso de la investigación científica. México: Limusa.

Toboso, J. (2004). Evaluación de habilidades cognitivas en la resolución de problemas matemáticos. Tesis doctoral, Universidad de Valencia. [Documento en línea]. Disponible: http://www. tdx.cat/TDX-0519105-125831. [Consulta: 2009, Marzo 21]

UPEL (2003) Manual de trabajo de grado de especialización y maestría y tesis doctorales. Venezuela: FEDUPEL.

Vergnaud, G. (1982) A classification of cognitive tasks and operations of thought involved in addition and subtraction problems. In carpenter, T.; Moser, J. \& Romberg, T. (1982). Addition and subtraction. A cognitive perspective. Hillsdale, N. J.: Lawrence Erlbaum. Pp. 39-59.

Vergnaud, G. (1983 a). Quelques problèmes de la didactique a propos d'un example: les structures additives. Atelier Internacional d'Eté: Recherché en Didactique de la physique. La londe les Maures, Francia, 26 de Junio a 13 de Julio.

Vergnaud, G. (1983 b). Multiplicative structures. In Lesh, R. and Landau, M. (Eds.) Acquisition of Mathemtics Concepts and Processes. New York: Academic Press Inc. Pp. 127-174.

Vergnaud, G. (1988). Multiplicative structures. In Hiebert, H. and Behr, M. (Eds.). Research Agenda in Mathematics Education. Number Concepts and Operation in the Middle Grades. Hillsdale, N. J.: Lawrence Erlbaum. Pp. 141-161.

Vergnaud, G. (1990). La théorie des champú conceptuels. Recherchés en Didactique des Mathématiques. 10 (23) : 133-170.

Vergnaud, G. Et al. (1990). Epistemology and psychology of mathematics education. In Nesher, P. \& Kilpatrick, J. (Eds.) Mathematics and cognition: A research systhesis by International Group for the Psychology of Mathematics Education.
Cambridge: Cambridge University Press.

Vergnaud, G. (1993). Teoría dos campos conceituais. In Nasser, L. (Ed.) Anais do $1^{\circ}$ Seminario Internacional de Educacao Matemática do Rio de Janeiro. P. 1-26.

Vergnaud, G. (1994). Multiplicative conceptual field: what why? In Guershon, H. And Confrey, J. (1994). (Eds.) The development of multiplicative reasoning in the learning of mathematics. Albany, N. Y.: State University of New York Press. Pp. 4159.

Vergnaud, G. (1996 a) . Education: The best part of Piaget's heritage. Swiss Journal of Psychology, 55(2/3): 112-118.

Vergnaud, G. (1996 b). A trama dos campos conceituais na construcao dos conhecimentos. Revista do GEMPA, Porto Alegre, N| 4: 9-19.

Vergnaud, G. (1997). The nature of Mathematical concepts. In Nunes, T. \& Bryant, P. (Eds.) Learning and teaching Mathematics, and international perspective. Hove (Eadt Sussex), Psychology Press Ltd.

Vergnaud, G. (1998). A comprehensive theory of representation for Mathematics Education. Journal of Mathematical Behavior, 17(2): 167-181. 